\title{
Development of a Low-Cost, High Accuracy, Flexible Panel Indexing Cell with Modular, Elastic Architecture
}

\author{
Robert Brownbill( $^{(\mathbb{})}($ ID and Adam Roberts (D) \\ Electroimpact UK Ltd., Hawarden, UK \\ \{robertb, adamr\} @electroimpact.com
}

\begin{abstract}
The global aerospace industry is driving a demand for flexible manufacturing systems to accommodate multiple programs with variable capacities within a modular, economical production cell [1]. Traditional manufacturing cells often involve bespoke, monolithic hardware limited to single program use. This inherent restraint results in significant incurred costs and program disruption when reacting to design and capacity changes. This paper describes the development of a reconfigurable panel-indexing cell with a dynamic cost architecture as an alternative approach to established, monolithic tooling structures.
\end{abstract}

Keywords: Flexible manufacturing $\cdot$ Modular architecture $\cdot$ Aerospace panel indexing $\cdot$ Reconfigurable tooling

\section{Introduction}

This paper narrates the mechanical design thought-processes of this fixture's development. The best-practice approach discussed, champions the move away from highcomplexity, high-cost, bespoke engineering solutions towards a design philosophy maximizing the use of commercially available off-the-shelf components to reduce cost and lead-time. In suitable applications, the reduction in capital expenditure and expedited time to enter production surpass the associated disadvantages. This reversal in engineering mindset should prove lucrative across all engineering disciplines.

Electroimpact are a world-leading supplier of automation and tooling systems for the aerospace industry; supplying equipment and solutions to all of the major aerospace manufacturers across the globe.

In addition to the supply of industrial solutions, Electroimpact (EI) are increasingly prioritising the collaboration of manufacturing research directly with industry and through partnerships with research and academic institutions. Such collaborations have resulted in the development of industry leading technologies, most notably that of Automated Fibre Placement (AFP) [2] and Accurate Robot Technology [3].

More recently, a growing demand for flexible, elastic manufacturing systems has been observed. The drivers behind this demand are primarily rate flexibility, programme flexibility and modular architecture. 
During the development phase of the Clean Sky 2, VADIS collaboration program [4], EI identified the opportunity to incorporate the indexing and metrology requirements alongside flexible, modular architecture, allowing precise indexing capability within a single, configurable tooling solution.

\section{Objectives}

\subsection{Innovative Design Methodology}

The design methodology used for this fixture was to reduce costs as far as practicable without compromising on accuracy, rigidity or functionality. This was done through the extensive use of commercially available off-the-shelf components from manufacturers. This is a reversal of the mindset from the 1990s/2000s where added complexity was seen to be the way forward.

This price reduction thus enables a greater target market for the fixtures, enables more budget to be spent on other areas such as increased automation and facilitates automation avenues into lower-rate aircraft programs.

The use of an mass-produced metrology system to set the tooling points opposed to a jig-integrated PLC controller is more cost-effective as not only is a typical laser tracker around half the price of a PLC control and servo system, the metrology equipment may be utilized in other areas of the facility between uses. A semi-skilled operator is required to use the metrology system and the fixture positioning will have a slight increase in setup time. However, these limitations are far outweighed for low to medium-rate aircraft programs by the initial capital savings.

\subsection{Functional Requirements}

The initial requirement of the VADIS panel jigs in the early development phase was to accurately index and clamp the upper and lower covers in nominal form during the scanning operation of the key datum features (the final metrology solution being the Nikon MV331). With the context of this straightforward remit, initial concepts showed conventional tooling solutions with interchangeable indexing profiles, Fig. 1.

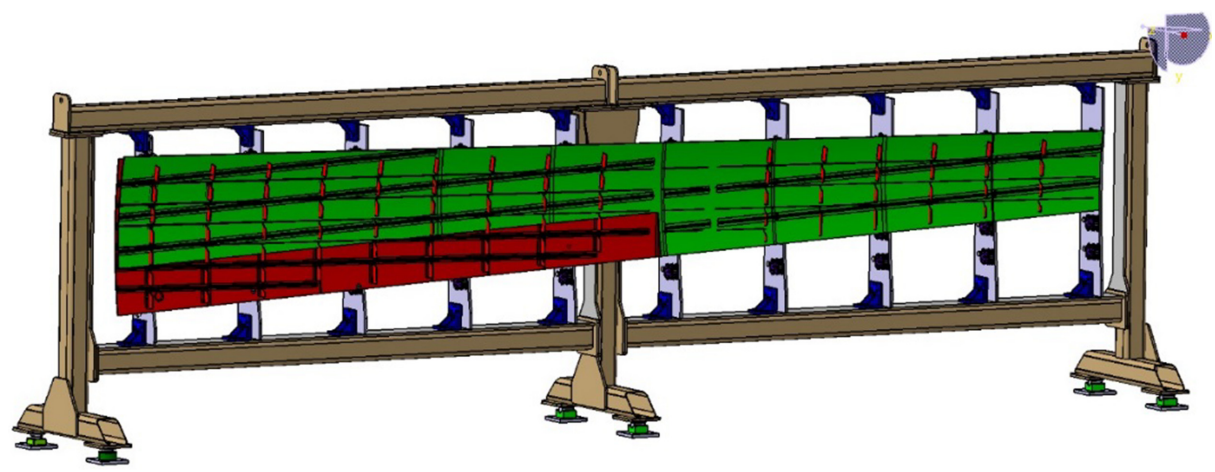

Fig. 1. Typical panel indexing concept using traditional rigid tooling methods 
As the component design development progressed, it quickly became clear that a more flexible solution would be required to accommodate a broader selection of panel sizes. The design requirements ultimately converged to the following key criteria:

- Three upper panel assemblies indexed and clamped to nominal form:

- Mid-Scale Upper Test Panel - $5 \mathrm{~m} \times 1.4 \mathrm{~m}$

- Inboard Upper Panel - $5 \mathrm{~m} \times 1.3 \mathrm{~m}$

- Outboard Upper panels - $4 \mathrm{~m} \times 1 \mathrm{~m}$

- High locational accuracy of indexing features with minimal tolerance stack between metrology and contact features

- Integrated metrology reference system for both index setting and for laser radar positional reference

- High structural and thermal stability

- Controlled, measured clamping forces

\subsection{Commercial and Schedule Constraints}

Following on from the functional requirements and taking into consideration the constraints of both the funding stream and the extended development time, EI expanded the design objectives to include:

- Manual adjustment of panel configuration (using a Laser Tracker)

- Prioritisation the use of standard purchased components

- Development a low-cost prototyping platform for the load cell monitoring

\subsection{Modular Architecture}

Once the functional and commercial requirements had been identified, EI realised the opportunity to integrate modularity and scalability within the tool design with the following aims:

- Modular base design able to function as a stand-alone jig or as a multi-base cell

- Upgradeability of drive systems allow future adaptation for semi-automated panel configuration

\subsection{Cost Analysis}

The following cost analysis is a rough order of magnitude (ROM) comparison between bespoke PLC-integrated fixtures and the low-cost altern ative discussed within the latter sections of this paper. Also included are scalability costs to double the space envelope. 


\begin{tabular}{|c|c|c|}
\hline $\begin{array}{l}\text { Tooling Philosophy } \\
\text { (Comparable Working Volume) }\end{array}$ & $\begin{array}{l}\text { Cost* } \\
(G B P)\end{array}$ & $\begin{array}{l}\text { Lead Time } \\
\text { (Months) }\end{array}$ \\
\hline $\begin{array}{l}\text { - Bespoke, semi-automated flexible panel indexing jig. } \\
\text { - Integrated PLC control. } \\
\text { - Servo driven actuators. } \\
\text { TOTAL } \\
\text { - Additional cost to double space envelope. }\end{array}$ & $\begin{array}{l}£ 700 \mathrm{k}-900 \mathrm{k} \\
£ 250 \mathrm{k}-400 \mathrm{k}\end{array}$ & $12-18$ \\
\hline $\begin{array}{l}\text { - Low-Cost, manually configurable, flexible panel indexing jig } \\
\text { - Integrated PLC position feedback. } \\
\text { TOTAL } \\
\text { - Additional cost to double space envelope. }\end{array}$ & $\begin{array}{l}£ 400 \mathrm{k}-\mathbf{6 5 0} \mathrm{k} \\
£ 200 \mathrm{k}-300 \mathrm{k}\end{array}$ & 9-14 \\
\hline $\begin{array}{l}\text { - Low-Cost, manually configurable, flexible panel indexing jig } \\
\text { - Commercially Available Laser Tracker. } \\
\text { TOTAL } \\
\text { - Additional cost to double space envelope }\end{array}$ & $\begin{array}{l}£ \mathbf{3 0 0} \mathbf{k}-\mathbf{4 5 0} \mathrm{k} \\
£ 100 \mathrm{k}-200 \mathrm{k}\end{array}$ & 4-10 \\
\hline
\end{tabular}

* Correct ROM costs as of January 2020

\section{Architecture}

\subsection{Overview}

The modular concept of the jig's architecture lends itself to being easily reconfigurable. The concept consists of a fabricated steel base of a fixed length, the concept has chosen a nominal five metre base length. The base would be secured to the facility floor using industry standard, off the shelf, floor fixings. Each base would be capable of mounting up to four tooling posts.

A typical layout with three posts per base, Fig. 2, would be the recommended method. Current industrial applications see typical post spacing between one and two meters, to correspond with assembly feature locations. Owing to the module nature of the jig, several of these bases may be bolted together to create a larger panel cell, Fig. 3.

\subsection{Structure}

Both the bases and the posts are of welded fabrication from two-dimensional profiles, cut from steel sheet metal. Sacrificial material has been included in the weldment design such that the posts are machined, post fabrication, to add the precision features required for mounting the linear bearing rails, Y-drive supports and other tooling features. 


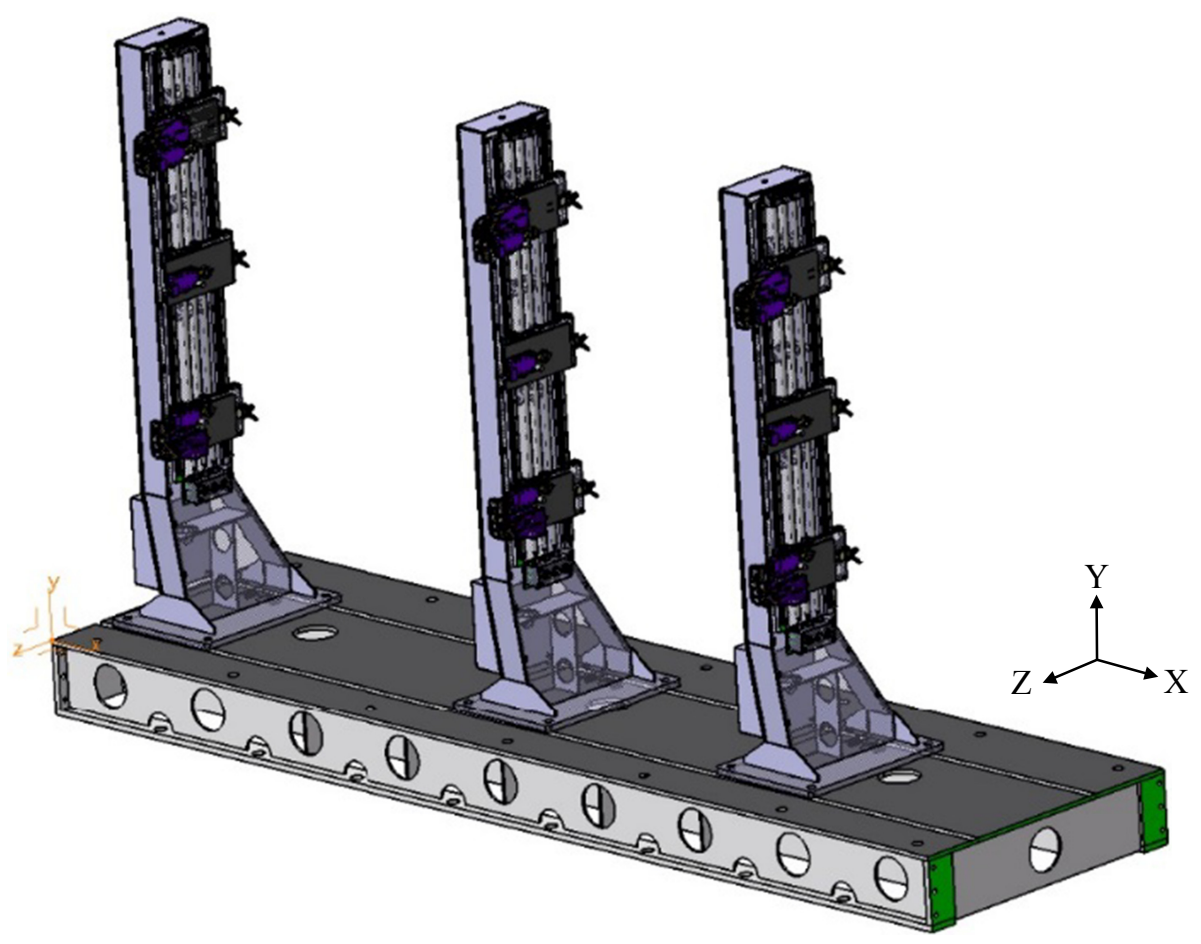

Fig. 2. Module base section with 3 tooling posts

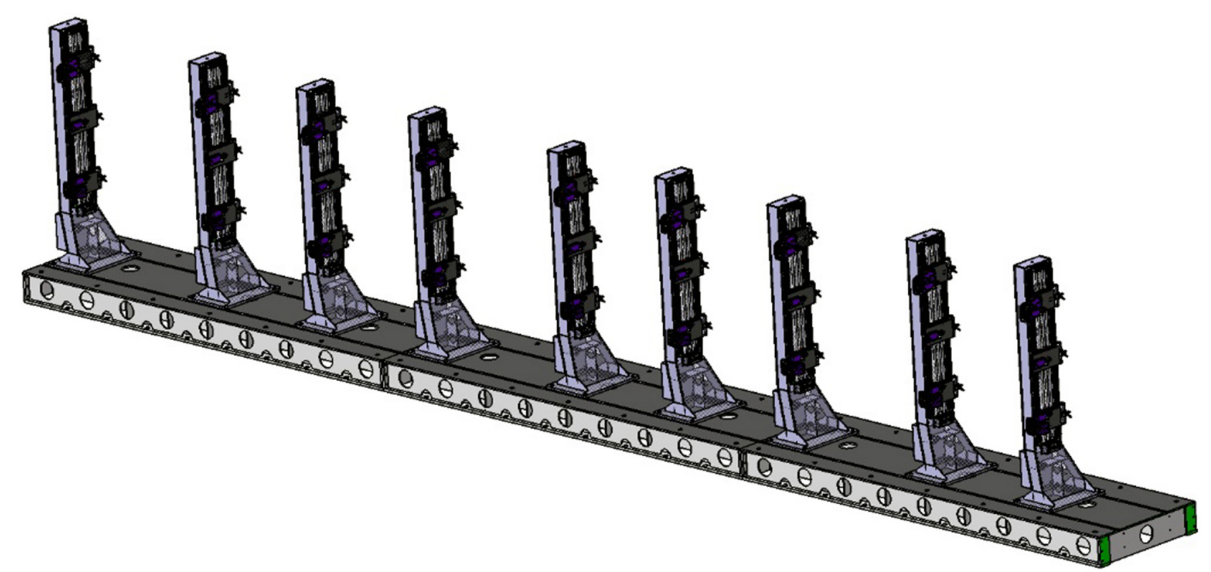

Fig. 3. Modular larger-scale application

The tooling posts are located to the base by means of T-bolts secured in T-slots. These longitudinal slots allow for the post X-position to be easily changed between applications by use of an overhead crane ( 2 lifting points are in the top of each post weldment). Positional tolerance of indexes in the span-wise, $\mathrm{X}$ direction, is typically an 
order of magnitude higher than the requirements for the cross-sectional profile indexes (Y \& Z directions). Although methods could be employed to enable high precision location of each post in the X-direction, the posts would be set typically with a laser tracker and positioned using the overhead crane with some small manual input.

The before-mentioned concept would be that as implemented for an industrial application. Within the research budget allocated to the project, VADIS fixture posts were mounted directly to the floor, Fig. 4. This was chosen as the final installation location was a facility which includes floor mounted T-Slots. The industrial concept assumes a flat, basic concrete facility floor.

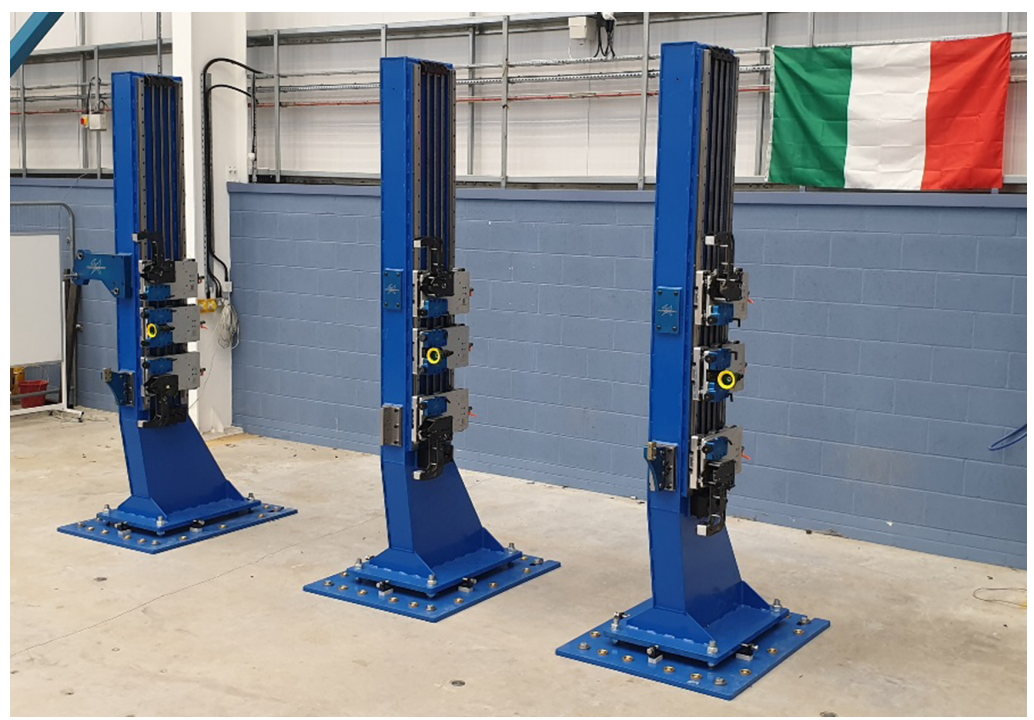

Fig. 4. VADIS fixture installed at electroimpact facility

The overarching function of the jig is to be flexible for a number of different panels configurations, Figs. 5, 6 and 7. The working envelope for the posts is a vertical range of $350 \mathrm{~mm}-1820 \mathrm{~mm}$ in $\mathrm{Y}$ and a horizontal range of $350 \mathrm{~mm}$ in Z. Post spacing can, of course, be varied in $\mathrm{X}$ to suit each application. A common post spacing was deemed suitable for the following three panels.

A thermal imaging camera was used on several occasions, in conjunction with a calibrated contact thermometer, to assess the thermal stability of the steel and aluminium components of the structure at several intervals during the day. It was found that on all occasions the jig temperature remained stable and uniform, Fig. 8, this was unless the external facility doors were left open for an extended period of time. It is therefore recommended that consideration be given to operations on the jig when any external doors are required to be open for extended periods. 


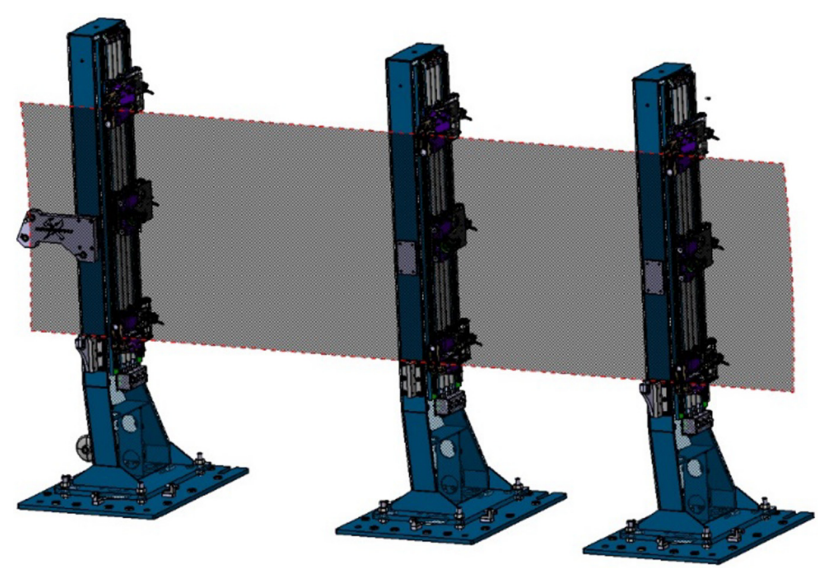

Fig. 5. Panel configuration 1

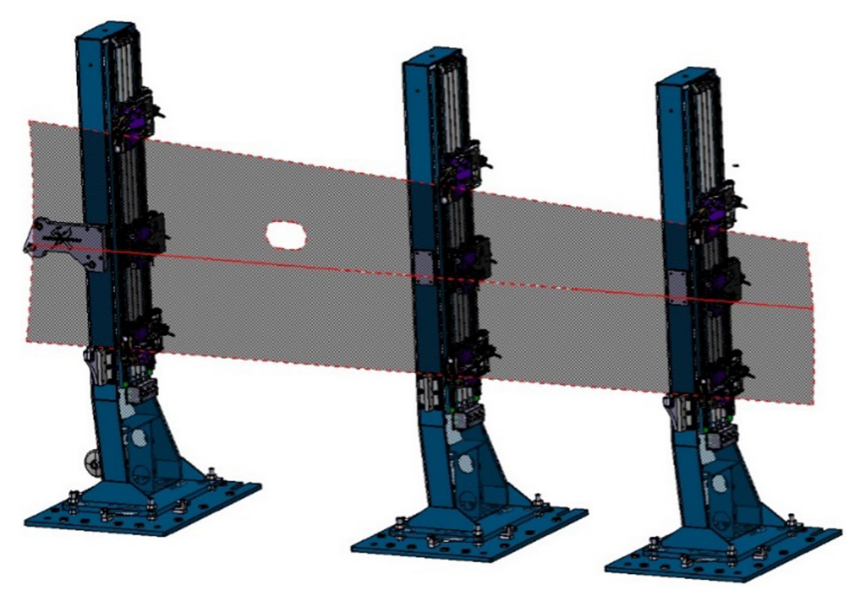

Fig. 6. Panel configuration 2

\subsection{Drive Systems}

One of the key objectives was to design the solution to be low-cost, as such, a high priority was placed on the use of off-the-shelf (mass produced) components. Not only is this highly beneficial in reducing costs but, should there ever be a need to replace or repair any of these components, the new parts can be on site in a matter of days. If they were to be of custom design, there would be the associated manufacturing lead time, typically in the order of four to eight weeks even for basic components. It was not possible to construct these assemblies entirely from bought out parts and as such, those 


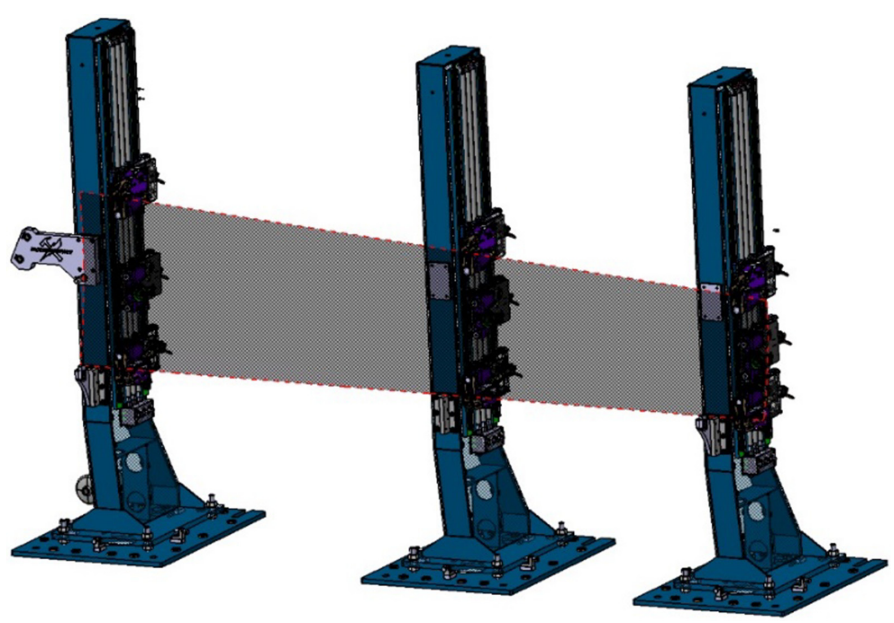

Fig. 7. Panel configuration 3

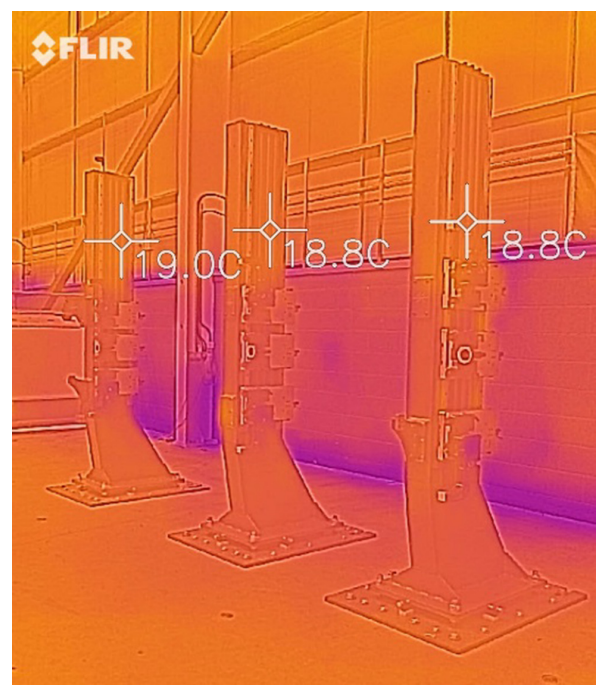

Fig. 8. Infra-red image of the VADIS Jig

custom parts were constrained to housings and non-wearing components. One example is the custom gearbox for the Y-drive, Fig. 9, although this is a custom machined 4axis part, this was able to be manufactured from aluminium reducing machining time and costs as the rotating components selected were all off-the-shelf industry standard bearings. 


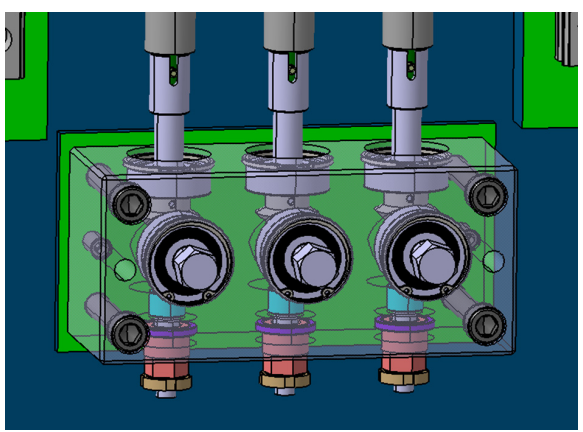

Fig. 9. Y drive gearbox

For the main Y-drive screws, it was chosen to select a trapezoidal thread form to reduce backlash as much as possible without the expense of ball screws. A bought-out screw was purchased and custom end operations added to interface with the gearbox spindle and the tensioning thrust bearing bracket.

Each of the three Y-drive screws allows adjustment of its corresponding Z-sled assembly, Fig. 10. The same utilisation of off-the-shelf drive components whilst minimising custom machined parts design methodology has been used here, two linear rails and trapezoidal drive screw assembly allow for adjustment in the Z-direction. These 2 degrees of freedom, coupled with the swivel index assembly allow the Optical Tooling Point (OTP) to be set to the panel

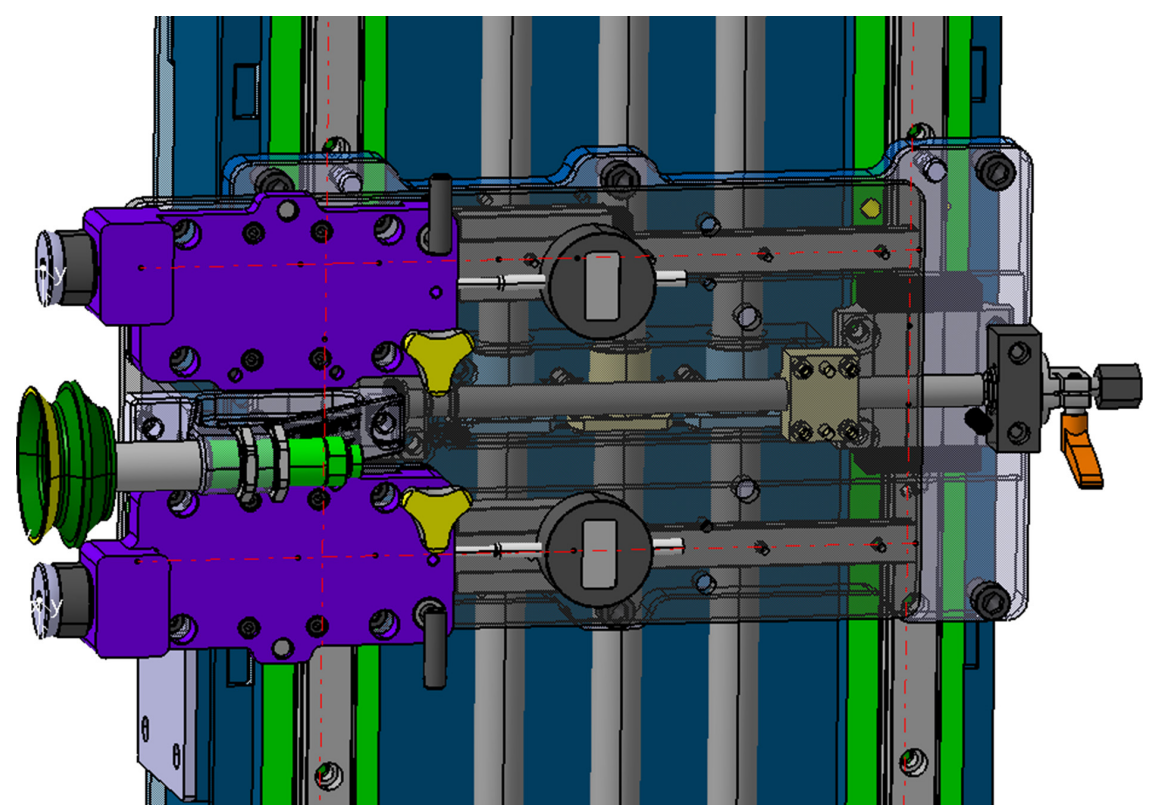

Fig. 10. Z-Sled indexing assembly 
For the Y-drive assembly, the thread pitch of the drive screws along with the tensioning thrust bearing at the top of the post and the weight of the sled create suitable friction in the drive system to prevent the sleds from moving without the need for an interlocking brake. Whereas, on the Z-sled assemblies, their smaller screw diameter and higher applied forces from the clamp/panel loads, it was decided that an off-the-shelf hand clamp, shown orange, Fig. 10, would be required to prevent unintentional movement and creep during operation.

All adjustments can be made by the use of readily available metric tools in any standard engineering tool kit. Both $\mathrm{Y} \& \mathrm{Z}$ adjustments can be done using a $17 \mathrm{~mm}$ or $19 \mathrm{~mm}$ spanner/socket either by hand, ratchet spanner or with the use of power tools with the correct socket drive attachment, Fig. 11.

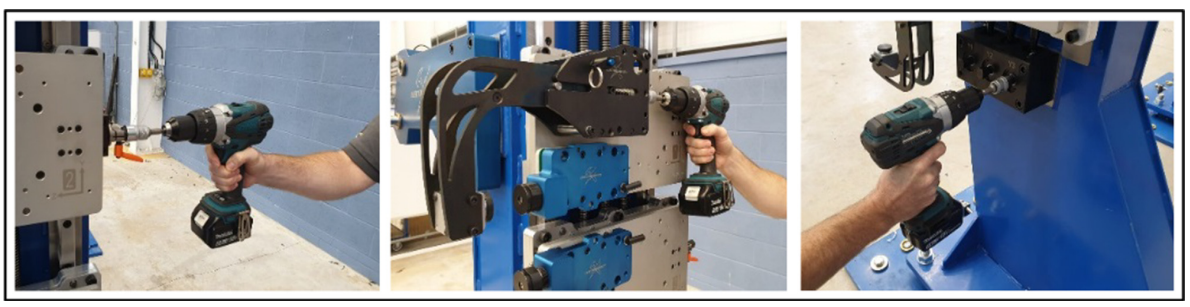

Fig. 11. Manual adjustment of the VADIS fixture with use of common power tools

\subsection{Indexing}

The swivel indexes used on this jig are of a tried and tested Electroimpact design. The cup and sphere method enables the indexing hemi-sphere to be removed and a Spherically Mounted Retroreflector (SMR) to be directly located into the cup, Fig. 12, retained with a magnet. The closer the SMR is to the final tooling contact surface, the smaller the tolerance stack up and the higher the accuracy of the system.

Although shown on a project specific, sliding assembly, Fig. 12, the actual swivel index mounts with a standard metric fine thread and is very compact assembly, Fig. 13.

As the SMR will set the centre point of the cup's semi-sphere, corresponding to the centre of the index hemi-sphere pad - this point only has to be on the surface of the part - no allowance needs to be made for the local angle of the panel at each location. The hemi-sphere can swivel through an angle of $20^{\circ}$ to allow for Y \& $\mathrm{Z}$ angular compliance, Fig. 14. 


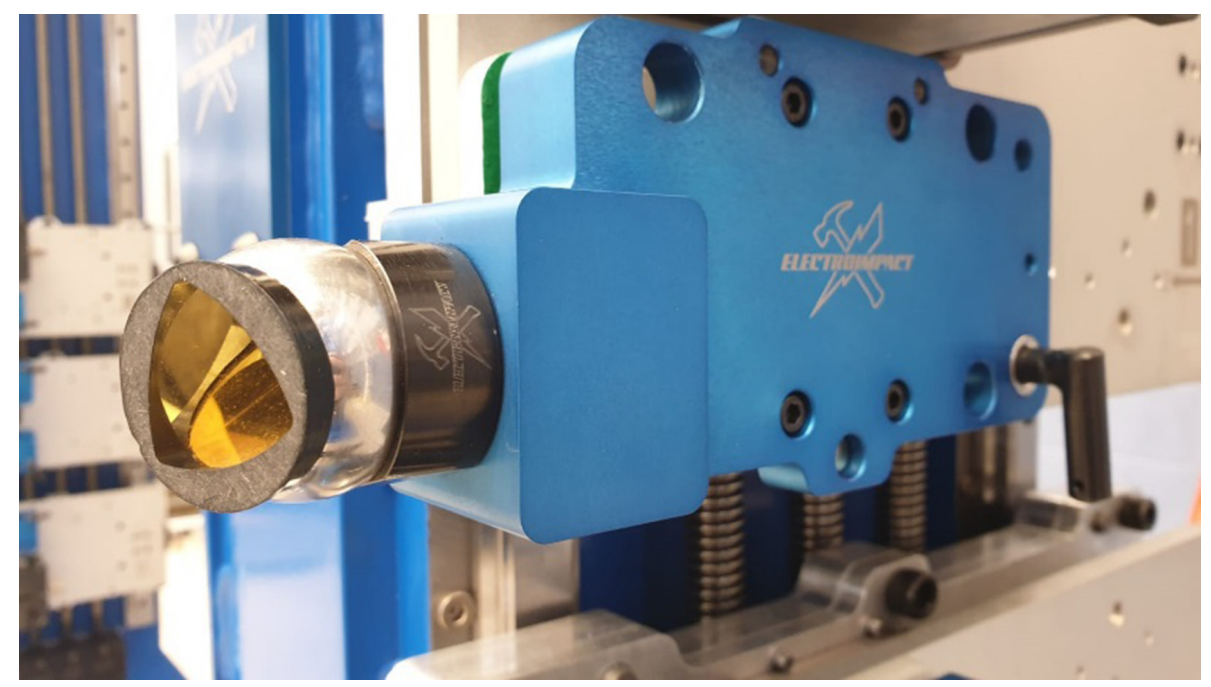

Fig. 12. Swivel index with SMR

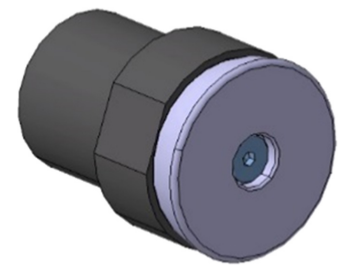

Fig. 13. CAD scheme of EI swivel index

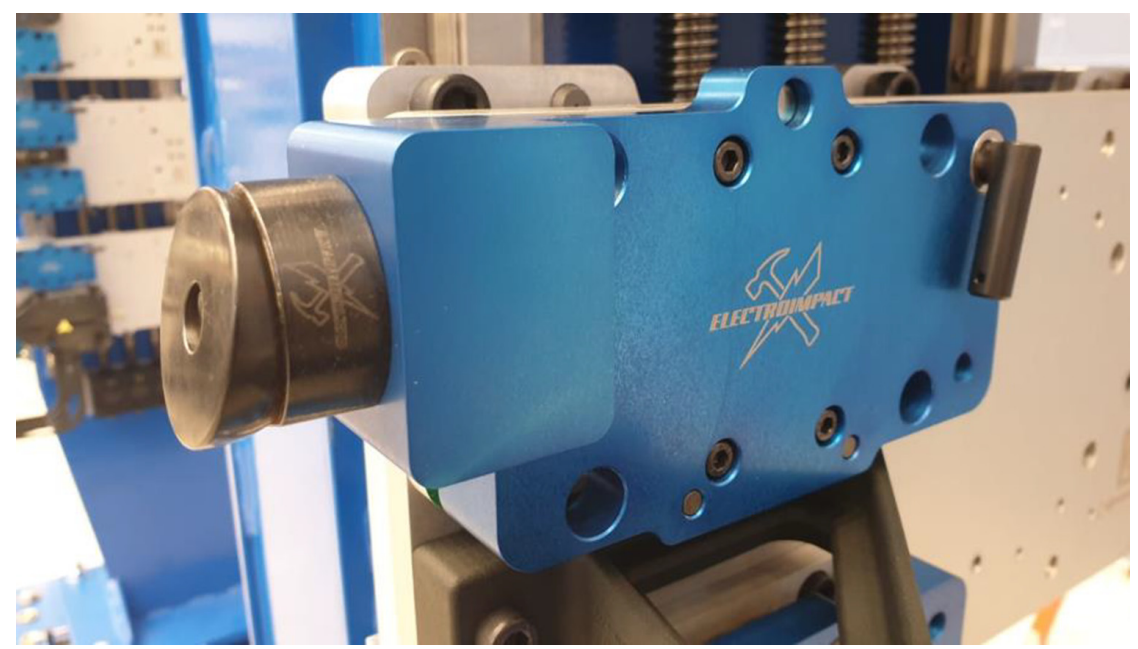

Fig. 14. EI swivel index assembly on the VADIS Jig 


\subsection{Metrology}

As with all precision tooling cells, a robust and highly accurate metrology architecture is required to facilitate the high precision setting of the optical tooling points. Limiting the tolerance stack up between the OTP and the contact surface greatly improves the accuracy and repeatability of the system. Where possible the use of metrology tooling has been removed. This has been achieved, as previously discussed, by enabling the SMR to be located directly into the Swivel Index nest and retained for setting. Another way of achieving this is to implement a similar scheme for the Jig Reference System (JRS). The JRS enables the laser tracker or metrology equipment to be located within the jig reference frame. The use of fixed nests, opposed to tooling holes requiring the use of a pin nest, reduce the tolerance stack up. These nests are screwed into the jig base and secured in place with high strength epoxy compound. An anodised aluminium ring with laser etched point ID numbers was put around these nests, Fig. 15, to easily identify the JRS points and protect the nests from damage during jig operation.

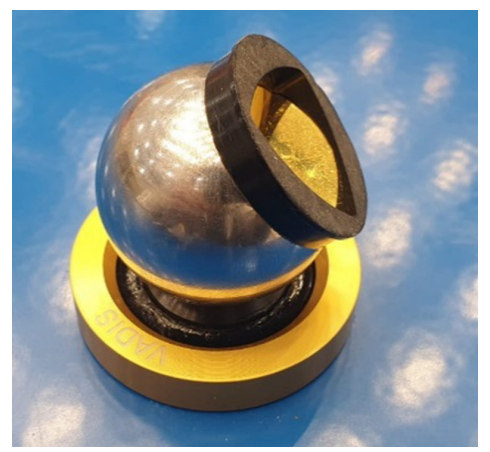

Fig. 15. Jig reference system nest and ID ring with SMR

To ensure a high quality JRS, the Unified Spatial Metrology Network (USMN) [5] feature in the Spatial Analyzer software was used to analyse the measurements of the actual JRS points from several stations. These point clouds were then run through the algorithm and scaled for temperature to obtain high accuracy values for the actual position of the JRS points. It took seven stations, Fig. 16, to ensure that each point was shot sufficient times to yield a reliable JRS valuation.

To set each of the swivel indexes the metrology operator would book into the JRS then, with the SMR positioned in the swivel index cup, use the $\mathrm{Y} \& \mathrm{Z}$ adjustability to precisely position the OTP. This process would be repeated for all OTPs on the jig. Although this process would take longer than an automated system with inbuilt absolute scales, a semi-skilled operator would be able to reconfigure the jig in a relatively short of time. It is currently estimated that with a competent operator the cell could be reconfigured with less than a thirty minutes disruption to production (for the VADIS fixture or similar). 


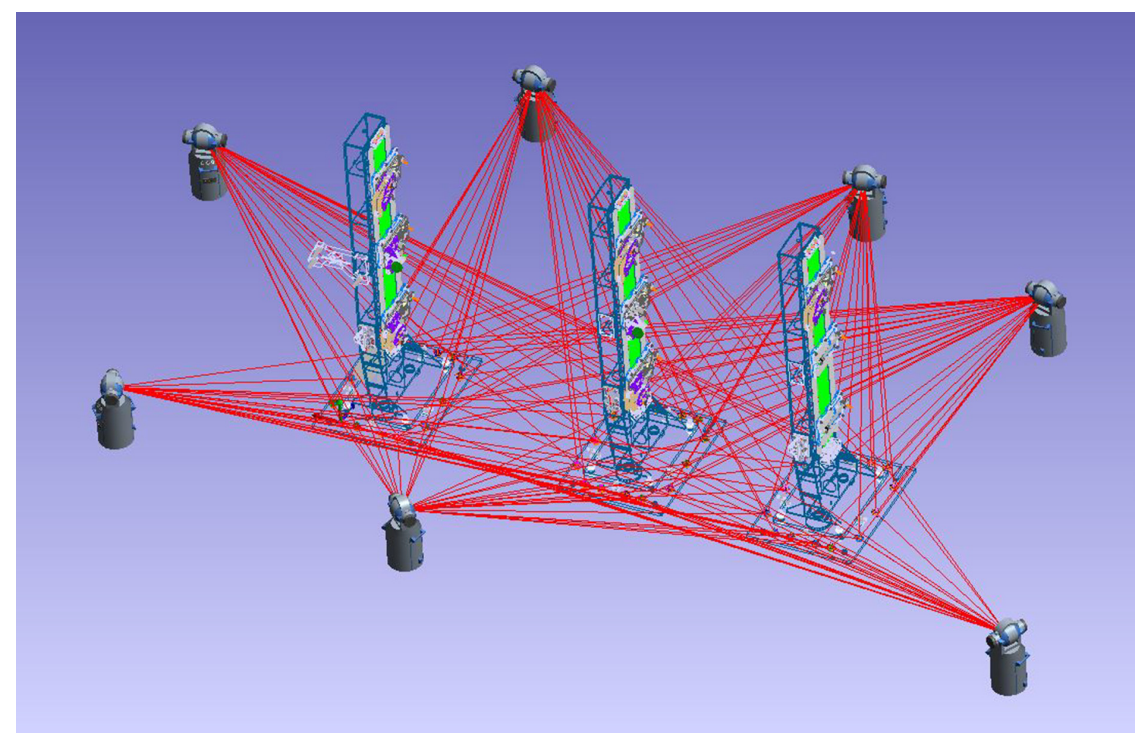

Fig. 16. USMN analysis of the VADIS Jig

Other benefits are such that the cost for the manually adjustable jig and the associated metrology system (laser tracker) are more cost-effective than a semi-automated cell with the added benefit that the metrology system is portable and can be used across multiple applications in the same facility.

\subsection{Force Sensing Clamps}

One of the key requirements from the Leonardo design department was for the application of a controlled, measured clamping force at predetermined locations across the panels.

EI's solution was a manually operated, screw-driven clamp assembly containing a $500 \mathrm{lb}-\mathrm{f}$ load cell directly behind the clamping pad, Fig. 17. To monitor the load cell values, a portable load cell amplifier was developed using a commercially available Arduino microcontroller, using a standard USB-B male-male interface cable.

Using a single interface across multiple load cells required the controller, Fig. 18, to be able to apply specific scaling factors to each individual load cell. Each clamp was given a unique identification (in this case, 'Upper Jig 1', 'Lower Jig 2' etc.) and is calibrated offline using measured calibrated masses. The scaling factors are then calculated and stored to the Arduino's internal non-volatile memory. 


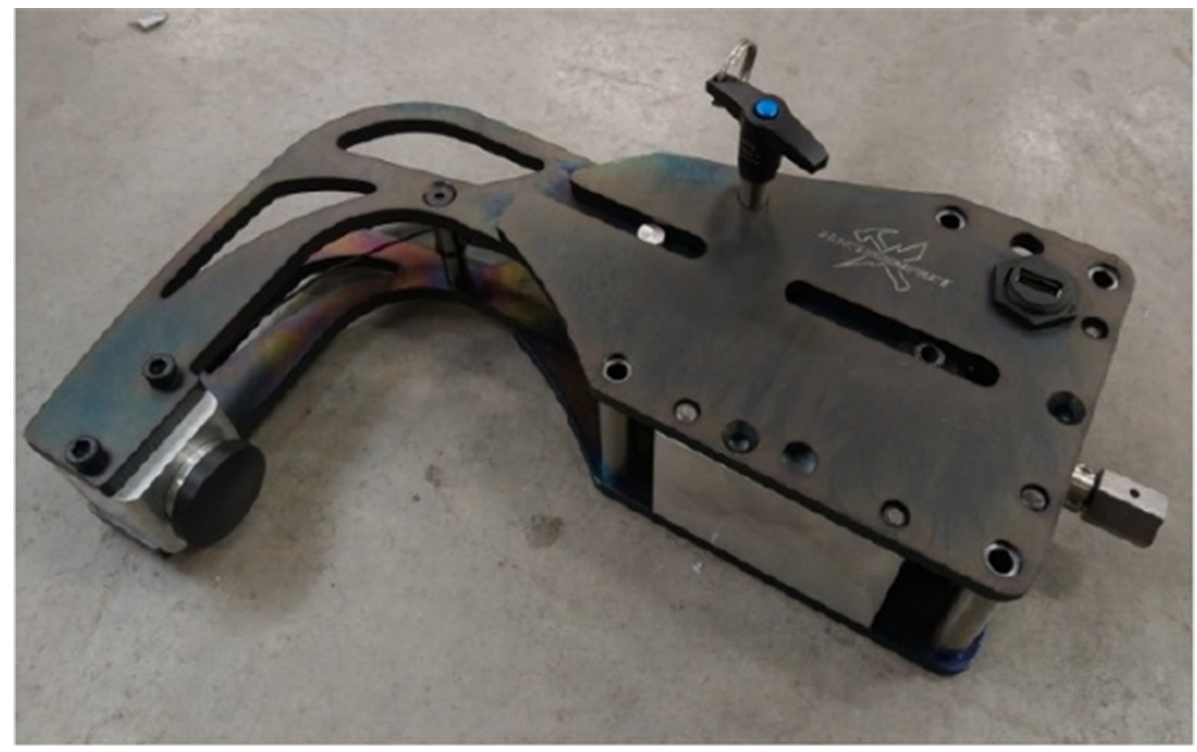

Fig. 17. Manual panel clamp with load cell feedback

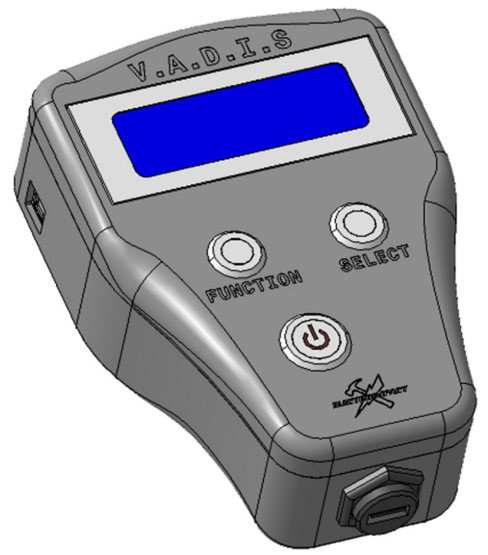

Fig. 18. VADIS hand pendant design

\section{Next Steps}

Following the successful implementation of the VADIS panel cell, EI intend to explore the subsequent developments:

Dynamic Cost Models. The jig architecture has been developed with scalability in mind. The configuration outlined within this paper is the most cost-effective solution, suitable for low rate with minimal configurations. EI propose the following levels of scalability: 
- Manual configuration of index and clamp locations - using portable laser tracker

- Semi-automated configuration using permanently mounted laser tracker and handheld HMI

- Semi-automated configuration using integrated absolute scales, central PLC control and handheld HMI

- Fully automated configuration using integrated servos for each drive and a central PLC control

Modular Panel Interfaces. This paper describes the use of swivel indexes in a modular sub-assembly to control panel contours. EI propose to develop interchangeable subassemblies to increase the range of indexing methods. Typical sub-assemblies would include stringer profile indexes, manhole clamping and datum hole pin locations.

Lateral Post Adjustment. One of the major limitations of the proposed jig is the lateral (Z) adjustment of the drive systems. EI propose to pursue an additional drive system within the post weldment base to allow lateral movement of the entire post assembly. This would increase the indexing envelope to include panels with significant sweep angles.

Non-contact Metrology Inspection. With the latest advancements of metrology hardware, high-accuracy non-contact measurement of key index locations is possible. This allows for remote measurement without the need for the operator to move an SMR to metrology features. Speeding up configuration time and whilst also allowing for in-process inspection of the component.

Smart Manufacturing. Within the development of flexible manufacturing systems is the desire for Smart Manufacturing - gathering relevant manufacturing process data and using advanced analytics to improve manufacturing efficiency [6]. EI have identified several features of the flexible jig which could be developed: recording of panel clamp load data, panel identification, panel in-jig process time, panel configuration time, pareto of the major planned and unplanned downtime.

Acknowledgements. This project has received funding from the European Union's Horizon 2020 research and innovation programme under grant agreement No 738221 . We are incredibly proud to work as part of this consortium. These developments would not have been possible without the hard work and support of Dr. Joseph Griffin, Dr. Konstantinous Bacharoudis, Dr. Alison Turner and Professor Svetan Rachev of the Centre for Aerospace Manufacture at the University of Nottingham along with Mr. Salvatore Cascella and his team at Leonardo Aircraft in Pomigliano d'Arco, Italy. 


\section{References}

1. Millar, A., et al.: Reconfigurable Flexible Tooling for Aerospace Wing Assembly, Aerospace Technology Conference and Exposition (2009)

2. Electroimpact Inc.: Composite Manufacturing. Electroimpact (2019). https://electroimpact. com/Products/Composites/Overview.aspx

3. DeVleig, R., et al.: Improved Accuracy of Unguided Articulated Robots. SAE International, Warrendale, PA (2009)

4. University of Nottingham Centre for Aerospace Manufacturing, Leonardo Aircraft, Electroimpact UK Ltd.: Variance Aware Determinate assembly Integrated System (VADIS), Nottingham, Pomigliano D'Arco, Hawarden (2019). https://cordis.europa.eu/project/id/738221

5. New River Kinematics: Unified Spatial Metrology Network, New River Kinematics (2018). https://www.kinematics.com/spatialanalyzer/usmn.php

6. Davis, J., et al.: Smart manufacturing, manufacturing intelligence and demand-dynamic performance. Comput. Chem. Eng. 47, 145-156 (2019)

Open Access This chapter is licensed under the terms of the Creative Commons Attribution 4.0 International License (http://creativecommons.org/licenses/by/4.0/), which permits use, sharing, adaptation, distribution and reproduction in any medium or format, as long as you give appropriate credit to the original author(s) and the source, provide a link to the Creative Commons license and indicate if changes were made.

The images or other third party material in this chapter are included in the chapter's Creative Commons license, unless indicated otherwise in a credit line to the material. If material is not included in the chapter's Creative Commons license and your intended use is not permitted by statutory regulation or exceeds the permitted use, you will need to obtain permission directly from the copyright holder.

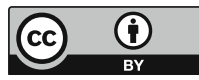

